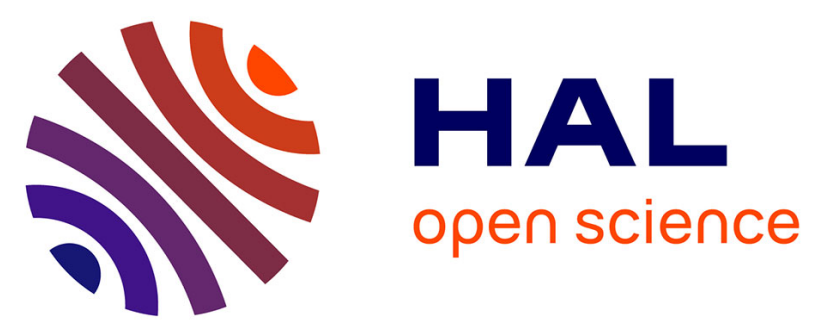

\title{
Fast and ergonomic extraction of adherent mammalian cells for NMR-based metabolomics studies
}

\author{
Manhal Mili, Baptiste Panthu, Anne-Marie Madec, Marie-Agnès Berger, \\ Gilles J P Rautureau, Bénédicte Elena-Herrmann
}

\section{- To cite this version:}

Manhal Mili, Baptiste Panthu, Anne-Marie Madec, Marie-Agnès Berger, Gilles J P Rautureau, et al.. Fast and ergonomic extraction of adherent mammalian cells for NMR-based metabolomics studies. Analytical and Bioanalytical Chemistry, 2020, 412 (22), pp.5453-5463. 10.1007/s00216-020-02764-9 . hal-03092830

\section{HAL Id: hal-03092830 \\ https://hal.science/hal-03092830}

Submitted on 3 Jan 2021

HAL is a multi-disciplinary open access archive for the deposit and dissemination of scientific research documents, whether they are published or not. The documents may come from teaching and research institutions in France or abroad, or from public or private research centers.
L'archive ouverte pluridisciplinaire HAL, est destinée au dépôt et à la diffusion de documents scientifiques de niveau recherche, publiés ou non, émanant des établissements d'enseignement et de recherche français ou étrangers, des laboratoires publics ou privés. 


\title{
Fast and Ergonomic Extraction of Adherent Mammalian Cells for NMR-based Metabolomics Studies
}

\author{
Manhal Mili ${ }^{1}$, Baptiste Panthu², Anne-Marie Madec², Marie-Agnès Chauvin², Gilles J.P. Rautureau, \\ Bénédicte Elena-Herrmann ${ }^{3 *}$ \\ 1 Univ Lyon, CNRS, Université Claude Bernard Lyon 1, ENS de Lyon, Institut des Sciences \\ Analytiques, CRMN FRE 2034, 5 rue de la Doua, 69100 Villeurbanne, France \\ ${ }^{2}$ Univ Lyon, CarMeN laboratory, INSERM, INRA, INSA, Lyon, Université Claude Bernard Lyon1, \\ 69121, Oullins, France \\ ${ }^{3}$ Univ Grenoble Alpes, CNRS, INSERM, IAB, Allée des Alpes, 38000 Grenoble, France
}

*Corresponding author: Benedicte Elena-Herrmann (benedicte.elena@univ-grenoble-alpes.fr)

ORCID:

Baptiste Panthu: 0000-0001-9430-4061

Gilles J.P. Rautureau: 0000-0002-1064-0293

Benedicte Elena-Herrmann: 0000-0002-0230-1590

Keywords: Metabolomics, NMR, adherent cells, extraction, quenching, metabolic profiles.

\section{ABSTRACT}

Cellular metabolomics has become key to elucidate mechanistic aspects in various fields such as cancerology or pharmacology, and is rapidly becoming a standard phenotyping tool accessible to the broad biological community. Acquisition of reliable spectroscopic datasets, such as Nuclear Magnetic Resonance (NMR) spectra, to characterize biological systems depends on the elaboration of robust methods for cellular metabolites extraction. Previous studies have addressed many issues raised by these protocols, however with little pondering on ergonomic and practical aspects of the methods that impact their scalability, reproducibility and hence their suitability to high-throughput studies or their use by non-metabolomics experts. Here, we optimize a fast and ergonomic protocol for extraction of 
metabolites from adherent mammalian cells for NMR metabolomic studies. The proposed extraction protocol, including cells washing, metabolism quenching and actual extraction of intracellular metabolites was first optimized on HeLa cells. Efficiency of the protocol, in its globality and for the different individual steps, was assessed by NMR quantification of 27 metabolites from cellular extracts. We show that a single PBS wash provides a seemly compromise between contamination from growth medium and leakage of intracellular metabolites. In HeLa cells, extraction using pure methanol, without cell scraping, recovered higher amount of intracellular metabolites than the reference methanol/water/chloroform method with cell scraping, with yields varying across metabolite classes. Optimized and reference protocols were further tested on eight cell lines of miscellaneous nature, and inter-operator reproducibility was demonstrated. Our results stress the need for tailored extraction protocols and show that fast protocols minimising time-consuming steps, without compromising extraction yields, are suitable for high-throughput metabolomic studies.

\section{INTRODUCTION}

Metabolomic approaches can provide extensive and instantaneous metabolic snapshots of ongoing biological processes in living systems. They are complementary to other omics strategies, and frequently used in studies related to human health. A wide range of samples, primarily biofluids, tissue and cells extracts, can be exploited for metabolomics studies. Biofluids are typically used to study metabolic consequences of pathophysiological processes on whole organisms as well as to identify biomarkers [1, 2], whereas tissue and cell samples are relevant to investigate mechanistic aspects [36]. Cellular metabolomics represents a cost-efficient and flexible approach, with good control of external variables, that is key to unravel essential aspects of cellular differentiation and proliferation [7], carcinogenesis [8, 9], or potential drug toxicity mechanisms [10]. While an increasing number of metabolomics studies on human adherent cell lines have been described over the past decade [11], preparation of cell extracts well suited for metabolomics studies still faces technical challenges as compared to the sampling of biofluids. Adequate protocols are not only crucial to ensure optimal quality and reproducibility of the analytical data, but they also govern accuracy of the metabolic portrait obtained for the observed biological system. Most evaluations of metabolite extraction procedures have been so far carried out for subsequent use of mass spectrometry coupled with either gas [12-16] or liquid chromatography [17-21] that result in metabolic detection windows and technical requirements, such 
as chemical derivatization or efficient protein background removal, well specific of these approaches. Few protocols have been developed towards Nuclear Magnetic Resonance (NMR)-based metabolomic analysis, and focused on selected steps of the overall extraction procedure [22-24].

Metabolite extraction from adherent cells consists in a series of entwined experimental steps that include washing of the cells to get rid of residue from culture media, quenching to stop metabolic activity, and cell harvesting and extraction of the intracellular metabolome using individual solvents or mixtures. Previous optimizations of metabolite extraction methods from adherent mammalian cell lines have selectively focused on cell washing $[12,17]$, harvesting $[13,25]$, quenching $[12,22]$ and extraction $[13$, $17,23]$, with little evaluation of their inter-connections and integration into the global procedure. In addition, protocols have been mostly exemplified on single cell lines, which differ across studies. Many questions therefore remain regarding the actual range of parameters that have significant effect on metabolite extraction yields.

Growth media used for mammalian cell culture have a rich composition to supply cells with nutrients required for maintenance, growth and proliferation. Media removal is a perquisite for cell extraction to avoid contamination of extracts by exogenous compounds. The fast kinetics of cell metabolism with respect to experimental manipulation leads to strong metabolic perturbations within span of seconds after medium removal $[26,27]$. Metabolites such as Glucose, ATP and ADP have turnover rates of millimolar per seconds [26, 28, 29], while others are volatile, or quickly degraded by external factors such as light exposure [27]. Meanwhile, leakage of metabolites from the intracellular medium into the washing solution may also affect metabolomic measures. Due to their small molecular size, metabolites are easily lost from cells by diffusing through cell membrane and are secreted in the washing solution. This is especially the case when the solution has different osmotic strength than the growth medium $[12,30]$. For this reason, PBS was shown to be a better washing solution than other commonly used solvents like water, even if metabolites loss is inevitable [17]. While the whole procedure may be carried out in a cold room to slow down metabolism and minimize thermal shock [31], this may turn out impractical and in practice the use of either room-temperature PBS or ice-cold PBS is often favoured, with a number of successive washes varying across studies $[13,15-17,19,22,23,25,32-34]$. In addition, metabolism still occurs until metabolite quenching which further changes metabolic profiles. Optimization and evaluation of protocols designed to avoid the contamination from exogenous growth media while minimizing metabolite loss prior to quenching therefore remains a timely issue. 
Metabolism quenching possibly stands as the most crucial step towards cellular metabolite extraction. Quenching aims at stopping enzymatic reactions, hence fix metabolite concentrations in the cells, and can be achieved using a variety of methods: freezing by liquid nitrogen [31], which is well adapted for tissue extractions $[35,36]$, addition of a quenching solvent discarded thereafter, mostly used for suspension cell cultures [37], or addition of a dual-role solvent that simultaneously quench and extract metabolites. For adherent mammalian cells, the latter method is most frequently privileged, as the easiest to implement, without loss of metabolites [15]. Treatment with cold methanol was shown to quench the metabolism and disrupt the cell membrane causing at the same time extraction of metabolites [22].

Carried out before or after quenching, cells harvesting aims at recovering the complete cellular matter from the culture dish. This is typically achieved either by cells scraping or trypsinisation. While scraping is performed on quenched cells, trypsinization is carried out prior to quenching and imposes strong physiological and mechanical stress through interaction of trypsin with membrane proteins to detach cells, and was shown to induce strong perturbations on the cellular metabolism [22]. Several studies have compared both strategies and have shown that scraping the cells achieved better extraction yield and weaker metabolite loss compared to trypsinization [13, 20,22]. Consequently, scraping has become the commonly used harvesting method over the past few years. Yet, it can lead to variable amounts of recovered cells between samples, especially when manipulating small diameter or fragile containers such as inserts, or when cells tend to form aggregates upon scraping. Kapoore et al. notably demonstrated that both trypsinization and scraping lead to metabolite loss that is dependent on cell type [15].

Extraction (stricto sensu) of metabolites is the last ensemble of steps that aims at recovering metabolites from the different cell compartments in a non-selective and non-destructive way. It is achieved using an extraction solvent and potentially further cell disruption by vortex or sonication. A wide variety of extraction methods were described for metabolomics studies [16, 22, 25, 38]. Pure methanol, aqueous methanol, or a mixture of methanol, water and chloroform (biphasic extraction) were shown to be reliable and efficient extraction blends leading to better extraction yields than other solutions such as perchloric acid or a mixture of water and acetonitrile $[23,39]$. However, contradictive reports are found regarding the efficiency of methanol or aqueous methanol extraction over the two-phase extraction protocol [13, 18, 20]. Biphasic extraction using methanol, chloroform and water has become arguably the most 
commonly used approach for cellular extraction. However, this method presents weaknesses associated with manipulation of toxic and volatile chloroform, and requires use of glassware little suited to high-throughput studies.

Overall practicality, and ergonomics in general, of existing protocols was given little pondering in previous studies. Yet, it impacts not only the feasibility of protocols in different laboratory settings, but also the more global accuracy, reproducibility and scalability of cell metabolic fingerprinting studies. As metabolomics has been rapidly democratizing as a standard tool for cell phenotyping, biologists face a recognizable need for generic, fast and efficient protocols, easily manageable by the non-metabolomics experts.

Here, we present a fast and ergonomic protocol for metabolite extraction from adherent mammalian cell cultures, tailored for in vitro NMR-based metabolomics studies and applicable to broader analytical strategies. The proposed method results from optimization of all washing, quenching and extraction steps, after evaluation of individual or combined sets of parameters. The protocol efficacy is assessed for eight different adherent mammalian cell lines, and its reproducibility is evaluated for HeLa cells by four different operators with reference to biphasic extraction. The proposed protocol comprises a limited number of simple steps, and balances efficient extraction of intracellular metabolites with minimum contamination from growth medium and minimum leakage of metabolites from the cells. It is fast, manageable without specific training, and easily automatable and scalable to small cell cultures in well plates for high-throughput studies. This optimized protocol aims at providing a suitable basis for sample preparation for broad metabolomic phenotyping characterization of central metabolism, across mammalian cell types.

\section{MAterials ANd Methods}

Materials. HeLa (Human cervical cancer cells), CACO2 (Human colorectal adenocarcimona cells), C2C12 (Mouse myoblasts), HEK 293 (Human embryonic kidney epithelial cells), 3T3-L1 (Mouse embryo fibroblasts), CHO-K1 (Hamster ovarian epithelial cells) were originally obtained from ATCC. INS-1E (Rat pancreatic $\beta$ cells) were a generous gift from Pr. P. Maechler (University Medical Center, Geneve, Switzerland) [40, 41]. HuH7 (Human hepatocellular carcinoma) cells were a gift from G. Mithieux's laboratory (Inserm U855, Lyon, France) [42]. 
Dulbecco's Modified Eagle's medium (DMEM) and RPMI were purchased from Gibco. Penicillinstreptomycin solution for cell culture and Foetal bovine serum (FBS lot BCBW7811) were purchased from Sigma-Aldrich. Dubelcco's Phosphate Buffered Saline (DPBS) 1x without calcium and without magnesium was purchased from Dominique Dutscher. Deuterium oxide $\left(\mathrm{D}_{2} \mathrm{O}, 99.8 \% \mathrm{D}\right)$ was obtained from Eurisotop. Lactate standard (TraceCERT ${ }^{\circledR}, 1000 \mathrm{mg} / \mathrm{L}$ ) for metabolite quantification was obtained from Sigma-Aldrich. All other reagents were of analytical grade.

Cell culture. INS-1E cells were cultured in RPMI-1640 culture medium containing $11 \mathrm{mM}$ glucose supplemented with $5 \%$ of heat-inactivated FBS, 2 mM glutamine, $10 \mathrm{mM}$ HEPES, $1 \mathrm{mM}$ Sodium Pyruvate, $100 \mathrm{U} / \mathrm{ml}$ penicillin, $100 \mu \mathrm{g} / \mathrm{ml}$ streptomycin and $50 \mu \mathrm{M}$ ß-mercaptoethanol (Sigma), at $37^{\circ} \mathrm{C}$ in a humidified atmosphere containing $5 \% \mathrm{CO}_{2}$. $\mathrm{HuH} 7$ cells were cultured in DMEM (1 g/L glucose) supplemented with $10 \% \mathrm{FBS}, 100 \mathrm{U} / \mathrm{ml}$ penicillin, $100 \mu \mathrm{g} / \mathrm{ml}$ streptomycin, at $37^{\circ} \mathrm{C}$ and $5 \% \mathrm{CO}_{2}$. The six other cell lines (HeLa, CACO2, C2C12, HEK 293, 3T3-L1, CHO-K1) were cultured in DMEM (4.5 g/L glucose, $2 \mathrm{mM}$ glutamax) supplemented with 10\% FBS, $100 \mathrm{U} / \mathrm{ml}$ penicillin, $100 \mu \mathrm{g} / \mathrm{ml}$ streptomycin, at $37^{\circ} \mathrm{C}$ and $5 \% \mathrm{CO}_{2}$. Cells were seeded in $10-\mathrm{cm}$ petri dishes to reach $80 \%$ confluence after 3 days to perform metabolome extraction.

Cell extraction and protocol optimization. For the recovering of metabolite extracts, cell plates were processed by batches of three for all critical steps (washing, quenching and extraction) and their optimisation, as a compromise between processing throughput by repetition of identical tasks, and minimisation of the global processing time for each sample. In this work, all steps for metabolite extraction were optimized sequentially: cells washing, quenching, harvesting, extraction. Evaluated protocols are further detailed throughout the results section. Extraction yields for 27 quantified metabolites were used to evaluate protocols efficiency. Optimizations of the different parameters for all extraction steps, and assessment of the protocol efficiency on multiple cell lines were conducted for at least three biological replicates for each condition and by the same operator to exclude experimental bias. Biphasic extraction using methanol, chloroform and water stands in this work as the "reference" protocol, detailed in Figure 1, and is adapted from the method described by Beckonert et al. for tissue extraction [36].

NMR Sample Preparation. After extraction, raw metabolite extracts were dried under a gentle $\mathrm{N}_{2}$ flow until complete evaporation, then stored at $-20^{\circ} \mathrm{C}$ until NMR sample preparation, right before analysis. $650 \mu \mathrm{l}$ of $\mathrm{D}_{2} \mathrm{O}$ phosphate buffer prepared as described in [36] were used to re-dissolve dried extracts by 
vortex for $30 \mathrm{~s}$. Extracts were then transferred to $1.5 \mathrm{ml}$ Eppendorf tubes and centrifuged at $13000 \mathrm{rpm}$ for 1 minute at $4^{\circ} \mathrm{C} .550 \mu \mathrm{l}$ of supernatant was transferred to $5 \mathrm{~mm}$ NMR tubes using a TECAN Evo 75 liquid handler.

NMR Spectroscopy. All NMR experiments were recorded on a Bruker Avance III spectrometer operating at $600.13 \mathrm{MHz}\left({ }^{1} \mathrm{H}\right.$ resonance frequency), equipped with a triple resonance $\mathrm{TCl}$ cryoprobe and cooled $\left(4^{\circ} \mathrm{C}\right)$ in a SampleJet sample changer. After automatic tuning and shimming, ${ }^{1} \mathrm{H}$ NMR profiles of cell extracts were acquired for each sample using a one-dimensional NOESY with gradients and water presaturation (pulse program noesygppr1d). The sample temperature was regulated at $27^{\circ} \mathrm{C}$. The relaxation delay was set to $3 \mathrm{~s}$, and the NOESY mixing time to $10 \mathrm{~ms}$. 128 transients were co-added, with an acquisition time of $2 \mathrm{~s}$ and a spectral width of $20 \mathrm{ppm}$. After Fourier transformation using an exponential line-broadening of $0.3 \mathrm{~Hz}$, spectra were manually phased, baseline corrected, and referenced to the glucose signal at $5.23 \mathrm{ppm}$ using Topspin (Bruker $\mathrm{GmbH}$, Germany). Two-dimensional experiments including ${ }^{1} \mathrm{H}$ JRES, ${ }^{1} \mathrm{H}-{ }^{1} \mathrm{H}$ TOCSY and ${ }^{1} \mathrm{H}-{ }^{13} \mathrm{C}$ HSQC were additionally recorded on a set of representative samples for metabolites annotations.

Metabolites quantification. Following metabolite identification (Table S1, ESI), metabolite concentrations in cell extract samples were exploited to evaluate step-by-step optimizations (washing, quenching, extraction parameters), as well as extraction protocols in their globality. Metabolite quantification was carried out from $1 \mathrm{D}^{1} \mathrm{H}$ NMR spectra. Relative quantifications were obtained by highthroughput integration of well resolved peaks corresponding to 27 metabolites using the Amix software (Bruker $\mathrm{GmbH}$ ) for each spectrum from all studied cell lines (total 224 spectra). A synthetic reference signal for quantification, defined from the ${ }^{1} \mathrm{H}$ spectrum of an external $3.36 \mathrm{mM}$ lactate standard, was inserted in each spectrum using the ERETIC 2 method [43] implemented in Topspin. Subsequently, interactive deconvolution of the NMR profiles using the Chenomx NMR Suite 8.2 (Chenomx Inc., Edmonton, Canada) provided a precise quantification of 41 metabolites in each sample for comparison of the optimized and reference extraction protocols on HeLa cells. Measured concentrations of metabolites in NMR samples range from a few micromolar to hundreds micromolar. Total quantity of matter was also evaluated by integration of the full spectrum to globally assess the extraction yield for each procedure.

\section{RESULTS}


The different steps for metabolite extraction from adherent mammalian cells were optimized sequentially after downsizing protocol variables according to previous literature described above. Tested conditions for HeLa cells extraction were systematically compared to the reference protocol (Figure 1a). All extracts were analysed by $1 \mathrm{D}{ }^{1} \mathrm{H}$ NMR spectroscopy for metabolite quantification. A representative spectrum with metabolite annotations in presented in Figure 2.

\section{Evaluation of washing methods.}

Variable volumes of warm PBS and number of washes were tested to assess contamination from residual growth media after its removal by pipetting: a single $3 \mathrm{ml}$ PBS wash, a single $6 \mathrm{ml}$ PBS wash, 2 washes with $3 \mathrm{ml}$ PBS each, a $6 \mathrm{ml}$ wash followed by a $3 \mathrm{ml}$ wash, and 3 washes with $3 \mathrm{ml}$ PBS. The different washing solutions were analysed by NMR spectroscopy after removal from the culture dish (Figure 3a). Remarkably, the total quantity of matter measured in the washes increases with both the total volume of PBS used and the number of successive washes, showing that metabolites may originate not only from residual growth medium, but also largely from leakage and secretion from the cells.

Since the timespan required to carry out multiple washes prior to quenching can reach minutes, we also examined intracellular metabolism kinetics on this timescale for a representative cellular nutrient (glucose) after growth medium removal. For this, we replaced the growth medium with a glucosedepleted medium before carrying out cellular extraction (with full protocol). This allows to quantify intracellular glucose at different time points following medium replacement. Figure $\mathbf{3 b}$ shows a sharp decrease of intracellular glucose within one minute of medium replacement. After 1 minute of glucose deprivation (30" of culture in glucose depleted-medium +30 " for medium removal and washing prior to quenching), intracellular glucose level has dropped 5-fold compared to 30 " glucose deprivation. Intracellular glucose concentration reaches a low plateau after 1.5 minute glucose deprivation in the media. Such rapid time-scale implies that multiple time-consuming washes prior to quenching should be avoided.

\section{Evaluation of quenching and extraction methods.}

In order to assess the possibility of disentangling the quenching and extraction steps in our protocol, we first evaluated a method proposed by Sellick et al. for the case of suspension-cultured cells [37]. The method uses $60 \%$ cold methanol supplemented with $0.85 \%$ (wt/vol) AMBIC, where AMBIC is tentatively 
recruited to protect cellular membranes, therefore blocking metabolism without emptying cells from their metabolite content. After quenching, this solution is to be discarded. Here, we recovered the mixture to determine the quantity of metabolite leaked into the quenching solution. Our data show a clear loss of metabolites using this approach (Figure S1, ESI), with no practical advantage to use such separate quenching steps in the case of adherent cells. We stress that all quenching approaches intrinsically impact, to some extent, the integrity of cellular membranes. Hence, quenching and extraction should hardly be considered as possibly independent steps in the whole procedure. In the following, we used a combined quenching and extraction step by cold methanol.

We then evaluated the conventional method that includes, directly after quenching, harvesting cells by scraping the culture dish against a protocol that skips this step. We hypothesized that polar metabolites are in fact completely extracted into methanol (or aqueous methanol) during the quenching/extraction step, making manual recovery of the cell debris unessential. Three groups of samples were assessed and the corresponding results are presented in Figure 4. For the first group, $3 \mathrm{ml}$ methanol was spread across the dish by a tilting motion repeated three times, then transferred into a glass tube, without scraping. The dish was rinsed with $1 \mathrm{ml}$ methanol, and the solution pooled with the primary extract. For the second group, cells were detached from the dish using a cell lifter directly after addition of $3 \mathrm{ml}$ of cold methanol. The mixture of solvent and cell debris were then transferred into a glass tube, and the dish was rinsed with $1 \mathrm{ml}$ methanol for full recovery of cellular material. The third reproduced the same protocol with cell scraping using a methanol/water $3: 1(\mathrm{v} / \mathrm{v})$ solution as extraction solvent. Scraping the cells had no significant effect on the majority of metabolites (Figure 4), with increased yield for only 6 metabolites out of 27 when harvesting the cells with a lifter. These are mainly purine and $\left(5^{\prime}-5^{\prime}\right)-$ dinucleotides as well as myo-inositol and some derivatives of carboxylic acids (glutathione, creatine), present at concentrations less than $0.1 \mathrm{mM}$ in the cellular extracts. The combination of scraping with the use of aqueous methanol as an extraction solvent permitted to retrieve significantly higher concentrations for only 7 metabolites out of 27 than when using a simple extraction with methanol, without scraping. Glutamine was the most impacted metabolite.

We then further refined our assessment of extraction parameters, by probing altogether the nature of extraction solvent (methanol or methanol/water 3:1), the number of successive extractions $(1,2$ or 3 ), total contact time between extraction solvent and cells $(0,3$, or 6 minutes), and the total volume of extraction solvent ( 4 or $7 \mathrm{ml}$; volumes were chosen to allow complete coverage of the $10 \mathrm{~cm}$ dish at the 
initial quenching step). Corresponding results are summarized in Figure 5, and show that increasing the contact time between the extraction mixture and cells did not impact the extraction yield (Figure 5a). Figure $\mathbf{5 b}$ illustrate the sensitivity to extraction solvent volume, and fractionation of extraction. For a given total volume of extraction solvent, the use of successive extractions tended to increase extraction yields, however increasing the total volume of methanol from 4 to $7 \mathrm{~mL}$ did not have a positive impact. The use of a $3+1 \mathrm{~mL}$ methanol extraction gave optimal yields for 18 out of 27 metabolites.

From all the above observations regarding washing, quenching and extraction, together with considerations of protocol ergonomics and rapidity of execution, we defined an optimized protocol based on a simple methanol extraction without scraping (Figure 1). The proposed protocol was further evaluated in reference to conventional water/methanol/chloroform extraction by a detailed quantification of 41 extracted metabolites in Hela cells (Table S2 \& Figure S2, ESI), before assessment in different cell lines.

\section{Efficacy of the optimized protocol in eight cell lines.}

The optimized and reference protocols were compared for metabolite extraction in eight different cells lines (Figure S3, ESI). In seven cell lines (Hela, HuH7, INS-1E, C1C12, HEK 293, 3T3-L1, and CACO2), our fast (optimized) protocol showed excellent yields with respect to the reference, providing better or equivalent extraction for the majority of metabolites. Higher levels of amino-acids were in particular recovered. However, levels of acetate, formate, ethanol and nucleotides were overall increased in extracts following the reference method. In contrast, the optimized protocol showed a relatively poor performance for metabolite extraction in $\mathrm{CHO}-\mathrm{K} 1$ cells (ovarian epithelial cells).

\section{Intra- and inter-operator reproducibility of the optimized and reference methods.}

Intra- and inter-operator reproducibility assessment for both the optimized and reference extraction protocols was achieved by comparing HeLa extracts obtained by four different operators in quadruplicates. Intra- and inter-operator reproducibility may reflect the respective protocols' ergonomics. Samples were prepared by the different operators in the same laboratory, with identical consumables, in the span of a single day. When comparing measured metabolites concentrations between operators, we observed a comparable stability of the measures for most metabolites using the two protocols. A larger intra-operator variability is observed with the reference protocol for a number of metabolites, in particular acetate, isobutyrate, lactate, and myoinositol (Figure S4, ESI). We note that the standard 
deviation across replicates, one component of intra-operator reproducibility, is also an individual characteristics of the operator, with operator 2 remarkably providing more stable results than others for both protocols. Inter-operator reproductibility is higher in the case of the optimized protocol for almost all metabolites (Figure S5, ESI). These data overall emphasize the robustness of our proposed method.

\section{Discussion}

Evaluation of the endometabolome in mammalian adherent cells is naturally challenging, due to the structural diversity of cellular metabolites, their broad dynamic range, and cellular compartmentalization. Different cell lines display structural specificities in their membranes and given cellular functions associated with variable rates of enzymatic reactions that further complicates this task. Although no single method allows complete extraction of all classes of metabolites from mammalian cells, advanced knowledge on how extraction procedures may affect metabolite recovery is key to their optimization. Subsequently, extraction protocols may be modulated to suit the characteristics of specific cell lines and objectives of given metabolomic studies that either focus on pre-defined metabolite classes, or aim at untargeted evaluation of the broad intracellular metabolome.

Our data demonstrated that although eliminating growth media residues by washing is a necessary step to obtain meaningful endometabolome profiles, time remains a critical point. Metabolite depletion by active metabolism in the cell may have more deleterious impact than residual contamination from growth media, and time-consuming steps before quenching have substantial effects on metabolite extraction yields. We showed that a suitable compromise is achieved with a single step wash with warm PBS immediately followed by cold methanol quenching, in agreement with previous literature that showed a single PBS wash was less damaging than two consecutive washes $[12,44]$. We chose a PBS volume of $6 \mathrm{ml}$ to stronger dilute residual material from growth medium and minimize contamination as compared to a single $3 \mathrm{ml}$ wash. We note that regardless any efforts made to minimize pre-quenching delays, measured concentrations of metabolites in extracts cannot flawlessly reflect actual intracellular concentrations at the time of sampling, as the decrease of intracellular nutrients is most important during the first seconds after media removal. Measured metabolite levels can either under- or over-estimate true intracellular concentrations, due to leakage into the washing solution or conversion/degradation of metabolites by ongoing enzymatic reactions. Optimally, operators should manipulate one dish at a time 
to ensure that the quenching time-point remains minimal and constant, with pre-quenching steps contained within a $30 \mathrm{~s}$ timespan.

However, high-throughput studies require batch processing, and protocols suitable for automatization. For this reason, the specific added-value of manual cells scaping after quenching/extraction was investigated. Our data showed that most metabolites are suitably extracted by simple addition of methanol, without scraping, which paves the way for a fast, ergonomic and scalable protocol for cellular metabolite extraction. The absence of scraping also contributes to reproducibility of the procedure, and the presence of residual cellular material in the dishes after quenching/metabolite extraction allows further complementary use of this material, notably for imaging purpose (Figure S6, ESI). We note that despite efficient quenching properties, addition of methanol may generate artefacts, as described by Sauerschnig et al., and therefore proper storage of extracts at $-80^{\circ} \mathrm{C}$ is required immediately after extraction until measurement [45]. This is also important since incomplete quenching from partial denaturation of enzymes cannot be fully excluded [44].

Surprisingly, we found that large volumes of extraction solvents and increased number of successive extractions did not permit the recovery of a greater amount of quantified metabolites, and the use of 3 $\mathrm{ml}+1 \mathrm{ml}$ of solvent gave optimal results. Remotely from the actual extraction efficiency, this may be explained considering the drying step that precedes re-dissolution of extracts into the NMR buffer. Along this process, dry metabolite mixture is coated over the internal surface of the sampling tubes. For the largest volumes of extraction solvents, metabolites are therefore scattered over larger surfaces, which hinders subsequent re-dissolution into the small buffer volume (typically $<650 \mu \mathrm{l}$ ). To avoid loss of metabolites at this stage, the use of an extraction volume adapted to both the surface of the cell culture dish and the final re-dissolution volume of dried extracts is required. Gentle dissolution of dry residuals from top parts of the tubes by manual rocking throughout evaporation under $\mathrm{N}_{2}$ gas may also minimize metabolites loss.

Whereas the use of water in extraction mixture provides better extraction for some classes of metabolites (mainly nucleotides) as compared to pure methanol, addition of water considerably lengthens the drying of extracts by $\mathrm{N}_{2}$ gas, due to lesser volatility. The presence of water in the extraction solvent was also associated with greater variability between replicate measures for a number of metabolites such as myo-inositol, acetate, NADH, ATP+ADP, or AMP, while the use of pure methanol led to optimal intra- and inter-operator reproducibility. In the case of NAD+ and NADH, the individual 
values of concentrations alone are not of biological importance contrary to their ratio. Scraping the cells and using water in the extraction mixture induced a strong shift on the measured NAD+/NADH ratio, diverging from physiological values in the cell (Figure 4).

While the present study does not comparatively evaluate strategies for drying metabolite extracts, we stress that alternative methods to gentle evaporation under $\mathrm{N}_{2}$ gas flow can be employed, such as lyophilisation, or evaporation using a centrifugal vacuum concentrator. We note that availability of such devices in biology laboratories often governs the actual choice of drying method.

In the present study, we evaluated the efficiency of the reference and optimized extraction protocols in eight different cell lines, selected to cover diverse properties like tissue origin and function, main secreted metabolites classes, as well as size and membrane properties of the cells. Our results show the proposed optimized fast protocol provides efficient metabolite extraction for most cell lines, with the exception of $\mathrm{CHO}-\mathrm{K} 1$ cells. Comparative detailed performance of the pure methanol vs. biphasic extraction yet depends on cell lines, and may be associated with variable subcellular compartmentation, such as differentially developed endoplasmic reticulum, or the abundance of lipid storage in droplets. Ideally, extraction protocols should therefore be optimized for each cell line.

Overall, our optimized protocol represents a good balance between the simplicity of the method and the metabolite extraction yield. We reduced by half the number of steps from media removal to sample drying, as compared to biphasic reference extraction, while avoiding the use of toxic and volatile chloroform and manual cells scraping and separation of polar and organic phases, to the benefit of protocol rapidity, ergonomics and reproducibility. Whenever care must be taken for extraction of specific metabolite classes, such as nucleotides, minor adjustment the protocol such as individual addition of a scraping step or water in the extraction solvent may provide useful alternatives. This fast and ergonomic protocol to characterize the intracellular metabolome is also compatible with exometabolomic investigations of adherent cell lines. Altogether exo- and endo-metabolomes are relevant to elucidate mechanistic aspects of cellular physiopathology.

\section{CONCLUSION}

Adherent mammalian cell lines display wide differences in structure and membrane properties. They contain metabolites from different classes, with widely diverse properties of solubility, polarity, volatility, 
and chemical stability. While metabolite extraction procedures may be fine-tuned for each given cell line, general guidelines and protocols implementable by non-metabolomics experts are needed to promote accessibility of metabolomic investigations to the broad biological community.

Here, we demonstrated a fast and ergonomic protocol for adherent mammalian cell metabolite extraction towards NMR detection and quantification, by optimization of the pre-quenching, quenching and extraction steps. The proposed procedure minimises metabolite leakage, and simultaneously quenches metabolism and extracts a wide range of metabolites with a limited number of steps. It is easily implementable, scalable and automatable, and shown to be highly reproducible and effective for a broad range of cell lines. The protocol remains versatile as it can be easily modulated by minor modifications (addition of water in extraction solvent and/or optional cell scraping step) to meet specific needs for targeted metabolite studies. We promote the proposed methanol-based fast extraction procedure as a method of choice towards large-scale metabolomic studies in cells.

\section{Conflict of Interest}

The authors declare no conflict of interest.

\section{References}

[1] Zhang A, Sun H, Wang P, et al. Recent and potential developments of biofluid analyses in metabolomics. J Proteomics. 2012; 75: 1079-1088.

[2] Giskeødegård GF, Madssen TS, Euceda LR, et al. NMR-based metabolomics of biofluids in cancer. NMR Biomed. 2018; e3927.

[3] Zhang A, Sun H, Xu H, et al. Cell metabolomics. OMICS. 2013; 17: 495-501.

[4] Rinschen MM, Ivanisevic J, Giera M, et al. Identification of bioactive metabolites using activity metabolomics. Nat Rev Mol Cell Biol. 2019; 20: 353-367.

[5] Aranibar N, Borys M, Mackin NA, et al. NMR-based metabolomics of mammalian cell and tissue cultures. J Biomol NMR. 2011; 49: 195-206.

[6] Lefevre C, Panthu B, Naville D, et al. Metabolic phenotyping of adipose-derived stem cells reveals a unique signature and intrinsic differences between fat pads. Stem Cells Int. 2019; 2019: 1-16.

[7] Moussaieff A, Rouleau M, Kitsberg D, et al. Glycolysis-Mediated Changes in Acetyl-CoA and Histone Acetylation Control the Early Differentiation of Embryonic Stem Cells. Cell Metab. 2015; 21: 392-402.

[8] Li H, Ning S, Ghandi M, et al. The landscape of cancer cell line metabolism. Nat Med. 2019; 
25: $850-860$.

[9] Ortmayr K, Dubuis S, Zampieri M. Metabolic profiling of cancer cells reveals genome-wide crosstalk between transcriptional regulators and metabolism. Nat Commun. 2019; 10: 1841.

[10] García- Cañaveras JC, Castell J V., Donato MT, et al. A metabolomics cell-based approach for anticipating and investigating drug-induced liver injury. Sci Rep. 2016; 6: 27239.

[11] Hayton S, Maker GL, Mullaney I, et al. Experimental design and reporting standards for metabolomics studies of mammalian cell lines. Cellular and Molecular Life Sciences 2017; 74: 4421-4441.

[12] Kapoore RV, Coyle R, Staton CA, et al. Influence of washing and quenching in profiling the metabolome of adherent mammalian cells: A case study with the metastatic breast cancer cell line MDA-MB-231. Analyst. 2017; 142: 2038-2049.

[13] Dettmer K, Nürnberger N, Kaspar H, et al. Metabolite extraction from adherently growing mammalian cells for metabolomics studies: Optimization of harvesting and extraction protocols. Anal Bioanal Chem. 2011; 399: 1127-1139.

[14] Danielsson APH, Moritz T, Mulder H, et al. Development and optimization of a metabolomic method for analysis of adherent cell cultures. Anal Biochem. 2010; 404: 30-39.

[15] Kapoore RV, Coyle R, Staton CA, et al. Cell line dependence of metabolite leakage in metabolome analyses of adherent normal and cancer cell lines. Metabolomics. 2015; 11: 1743-1755.

[16] Hutschenreuther A, Kiontke A, Birkenmeier G, et al. Comparison of extraction conditions and normalization approaches for cellular metabolomics of adherent growing cells with GC-MS. Anal Methods. 2012; 4: 1953.

[17] Ser Z, Liu X, Tang NN, et al. Extraction parameters for metabolomics from cultured cells. Anal Biochem. 2015; 475: 22-8.

[18] Lorenz MA, Burant CF, Kennedy RT. Reducing time and increasing sensitivity in sample preparation for adherent mammalian cell metabolomics. Anal Chem. 2011; 83: 3406-3414.

[19] García-Cañaveras JC, López S, Castell JV, et al. Extending metabolome coverage for untargeted metabolite profiling of adherent cultured hepatic cells. Anal Bioanal Chem. 2016; 408: 1217-1230.

[20] Bi H, Krausz KW, Manna SK, et al. Optimization of harvesting, extraction, and analytical protocols for UPLC-ESI-MS-based metabolomic analysis of adherent mammalian cancer cells. Anal Bioanal Chem. 2013; 405: 5279-5289.

[21] Peterson A, Walker A, Sloan E, et al. Optimized Method for Untargeted Metabolomics Analysis of MDA-MB-231 Breast Cancer Cells. Metabolites. 2016; 6: 30.

[22] Teng Q, Huang W, Collette TW, et al. A direct cell quenching method for cell-culture based metabolomics. Metabolomics. 2009; 5: 199-208.

[23] Martineau E, Tea I, Loaëc G, et al. Strategy for choosing extraction procedures for NMR-based metabolomic analysis of mammalian cells. Anal Bioanal Chem. 2011; 401: 2133-2142.

[24] Belle JE Le, Harris NG, Williams SR, et al. A comparison of cell and tissue extraction techniques using high-resolution1H-NMR spectroscopy. NMR Biomed. 2002; 15: 37-44. 
[25] Muschet C, Möller G, Prehn C, et al. Removing the bottlenecks of cell culture metabolomics: fast normalization procedure, correlation of metabolites to cell number, and impact of the cell harvesting method. Metabolomics. 2016; 12: 151.

[26] de Koning W, van Dam K. A method for the determination of changes of glycolytic metabolites in yeast on a subsecond time scale using extraction at neutral pH. Anal Biochem. 1992; 204: 118-23.

[27] Villas-Bôas SG, Roessner U, Hansen MAE, et al. Metabolome Analysis: An Introduction. John Wiley and Sons; 2006.

[28] Rizzi M, Baltes M, Theobald U, et al. In vivo analysis of metabolic dynamics in Saccharomyces cerevisiae: II. Mathematical model. Biotechnol Bioeng. 1997; 55: 592-608.

[29] Schwiebert EM, Zsembery A. Extracellular ATP as a signaling molecule for epithelial cells. Biochim Biophys Acta - Biomembr. 2003; 1615: 7-32.

[30] Britten RJ, Mcclure FT. The amino acid pool in Escherichia coli. Bacteriol Rev. 1962; 26: 292.

[31] Kostidis S, Addie RD, Morreau H, et al. Quantitative NMR analysis of intra- and extracellular metabolism of mammalian cells: A tutorial. Anal Chim Acta. 2017; 980: 1-24.

[32] Danielsson APH, Moritz T, Mulder H, et al. Development and optimization of a metabolomic method for analysis of adherent cell cultures. Anal Biochem. 2010; 404: 30-39.

[33] Madji Hounoum B, Blasco H, Nadal-Desbarats L, et al. Analytical methodology for metabolomics study of adherent mammalian cells using NMR, GC-MS and LC-HRMS. Anal Bioanal Chem. 2015; 407: 8861-8872.

[34] Matheus N, Hansen S, Rozet E, et al. An easy, convenient cell and tissue extraction protocol for nuclear magnetic resonance metabolomics. Phytochem Anal. 2014; 25: 342-349.

[35] Zukunft S, Prehn C, Röhring C, et al. High-throughput extraction and quantification method for targeted metabolomics in murine tissues. Metabolomics. 2018; 14: 1-12.

[36] Beckonert O, Keun HC, Ebbels TMD, et al. Metabolic profiling, metabolomic and metabonomic procedures for NMR spectroscopy of urine, plasma, serum and tissue extracts. Nat Protoc. 2007; 2: 2692-2703.

[37] Sellick CA, Hansen R, Stephens GM, et al. Metabolite extraction from suspension-cultured mammalian cells for global metabolite profiling. Nat Protoc. 2011; 6: 1241-1249.

[38] Cao B, Aa J, Wang G, et al. GC-TOFMS analysis of metabolites in adherent MDCK cells and a novel strategy for identifying intracellular metabolic markers for use as cell amount indicators in data normalization. Anal Bioanal Chem. 2011; 400: 2983-2993.

[39] León Z, García-Cañaveras JC, Donato MT, et al. Mammalian cell metabolomics: Experimental design and sample preparation. Electrophoresis. 2013; 34: 2762-2775.

[40] Merglen A, Theander S, Rubi B, et al. Glucose Sensitivity and Metabolism-Secretion Coupling Studied during Two-Year Continuous Culture in INS-1E Insulinoma Cells. Endocrinology. 2004; 145: 667-678.

[41] Dingreville F, Panthu B, Thivolet C, et al. Differential Effect of Glucose on ER-Mitochondria $\mathrm{Ca} 2+$ Exchange Participates in Insulin Secretion and Glucotoxicity-Mediated Dysfunction of $\beta$ Cells. Diabetes. 2019; 68: 1778-1794. 
[42] Rieusset J, Fauconnier J, Paillard M, et al. Disruption of calcium transfer from ER to mitochondria links alterations of mitochondria-associated ER membrane integrity to hepatic insulin resistance. Diabetologia. 2016; 59: 614-623.

[43] Akoka S, Barantin L, Trierweiler M. Concentration measurement by proton NMR using the ERETIC method. Anal Chem. 1999; 71: 2554-2557.

[44] Lu W, Su X, Klein MS, et al. Metabolite Measurement: Pitfalls to Avoid and Practices to Follow. Annu Rev Biochem. 2017; 86: 277-304.

[45] Sauerschnig C, Doppler M, Bueschl C, et al. Methanol Generates Numerous Artifacts during Sample Extraction and Storage of Extracts in Metabolomics Research. Metabolites. 2017; 8: 1. 


\section{Figure Legends}

Figure 1: Schematic cell extraction protocols for NMR metabolomic investigation of adherent mammalian cells. For both reference (a) and optimized (b) methods, growth medium is first removed from the culture dish, before cells washing with $6 \mathrm{ml} \mathrm{PBS}$, and quenching with $3 \mathrm{ml}$ ice-cold methanol. For the conventional protocol (a) the dish is first rinsed with $1 \mathrm{ml}$ cold methanol, then cells are collected with a cell lifter and transferred into a glass tube, before $4 \mathrm{ml}$ chloroform and $3.6 \mathrm{ml}$ water are added to the cell suspension. After separation by gentle centrifugation ( $15 \mathrm{~min}$ at $4^{\circ} \mathrm{C}$ at $5000 \mathrm{rpm}$ ), the polar (i.e. top phase) and organic (bottom phase) fractions are collected individually. Our optimized protocol (b) includes a combined quenching and extraction step using methanol $(3+1 \mathrm{ml})$, without cell scraping. For both protocols, the obtained polar fractions are finally dried under a gentle $\mathrm{N}_{2}$ gas flow (alternatively, Iyophilisation or Speedvac can be used) until complete evaporation. Dried extracts are re-dissolved in $650 \mu \mathrm{l} \mathrm{NMR}$ buffer prepared as described by Beckonert et al. [36], vortexed for $30 \mathrm{~s}$, and centrifuged $\left(13000 \mathrm{rpm}, 4^{\circ} \mathrm{C}, 1 \mathrm{~min}\right)$ in a $1.5 \mathrm{ml}$ microtube. $550 \mu \mathrm{l}$ of the supernatant is then transferred into a $5 \mathrm{~mm}$ NMR glass tube for metabolic profiling.

Figure 2: Representative ${ }^{1} \mathrm{H}$ NMR spectrum $(600 \mathrm{MHz})$ for a HeLa cells extract with metabolite annotations, as obtained using our optimized protocol. Abbreviations: Bet: Betaine, ChoP: Phosphocholine, Glp: Pyroglutamate, GSH: Glutathione, NAC1: N-acetyl 1, MI: Myoinositol, PCr: Phosphocreatine, Pyr: Pyruvate, UDP-Gal: UDP-galactose, UDP-Glc: UDP-glucose, UDP-GIcNAc: UDP-N-acetyl-glucosamine

Figure 3: Sensitivity to washing parameters. (a) Total quantity of metabolites represented by total intensity of spectra in PBS washes recovered after washing the cells with different PBS volumes and number of washes; (b) Intracellular glucose concentration in the cells, as measured from extracts, after different times without contact with extracellular glucose. The total duration of pre-quenching steps is $30 \mathrm{~s}$, corresponding to cell washing (once) with $6 \mathrm{ml} \mathrm{PBS}$. The point at time zero in the graph represents a projected glucose concentration at the instant of media removal, not measurable. Error bars represent +/- standard deviation from 3 biological replicates. 
Figure 4: Metabolite concentrations in extracts as a function of cell harvesting method (with or without cells scraping) and solvent nature (methanol $3+1 \mathrm{ml}$, or methanol $3 \mathrm{ml}+$ water $1 \mathrm{ml}$ ). (a) Metabolites for which scraping after extraction with methanol or aqueous methanol does not significantly impact the concentration in extracts and (b) metabolites which concentrations are significantly impacted by scraping and/or extraction with water in addition to methanol. Six biological replicates were considered for each protocol; error bars represent + /- standard deviation. * significant variations between the two protocols (Student t-test p-values $<0.05$ ).

Figure 5: Sensitivity to extraction parameters (a) Overview of fold change for the concentration of metabolites extracted with a range of extraction procedures using different solvents for extraction and increasing contact times between solvents and cells, with reference to an extraction by twice $3 \mathrm{ml}$ methanol followed by $1 \mathrm{ml}$ methanol, and no waiting time; (b) Overview of fold change for metabolites extracted with different extraction procedures, varying total solvent extraction volumes and number of successive extractions. Three biological replicates were considered for each protocol. 


\section{Figure 1}

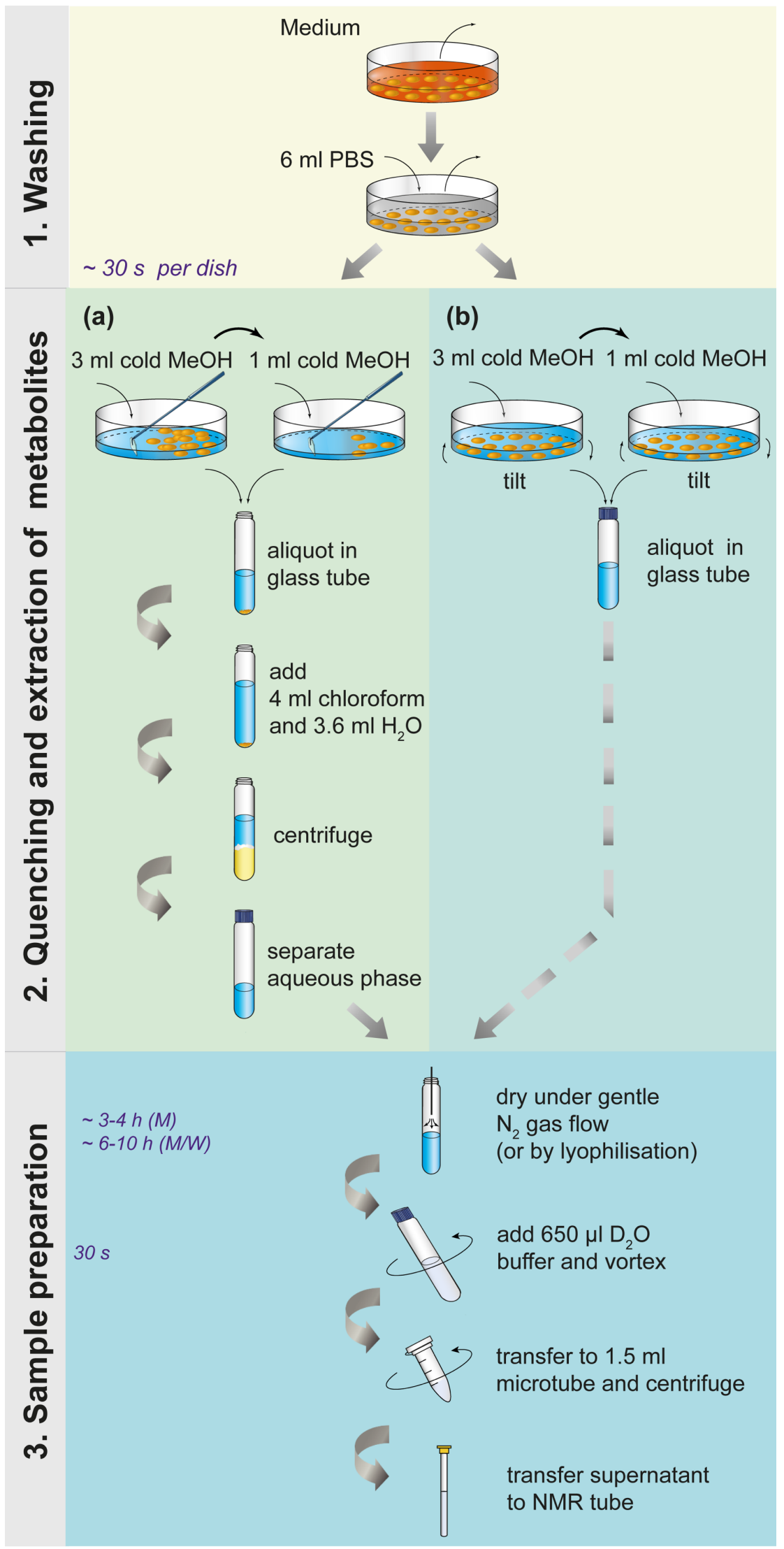


Figure 2

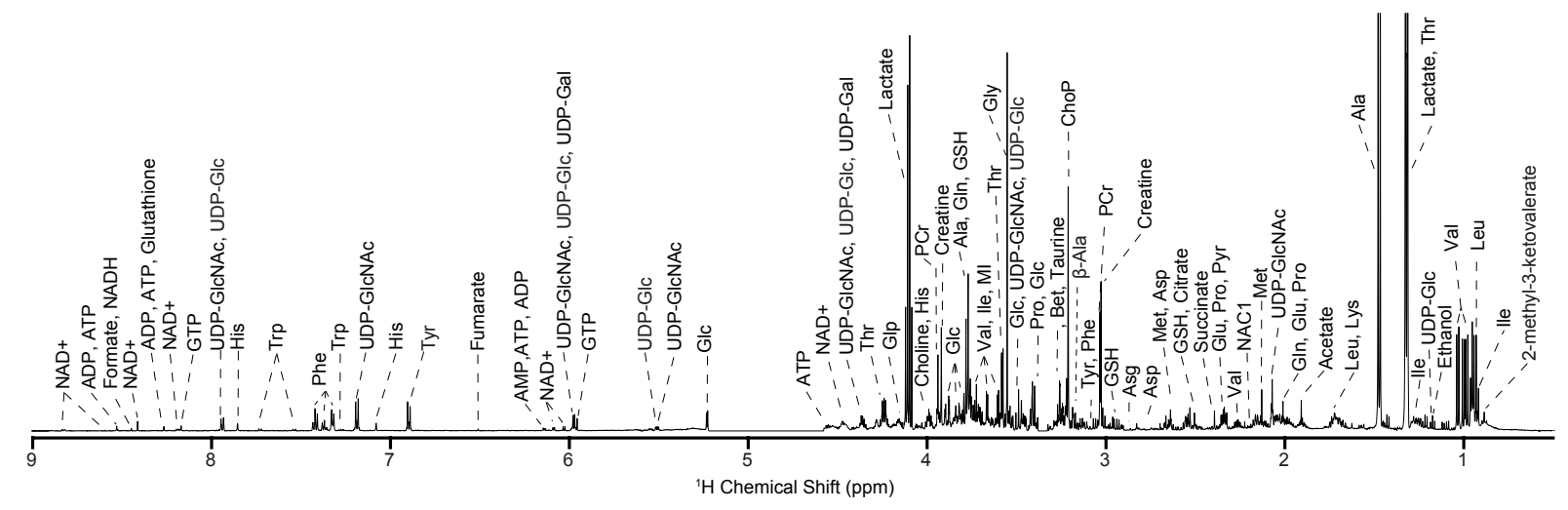

Figure 3

(a)

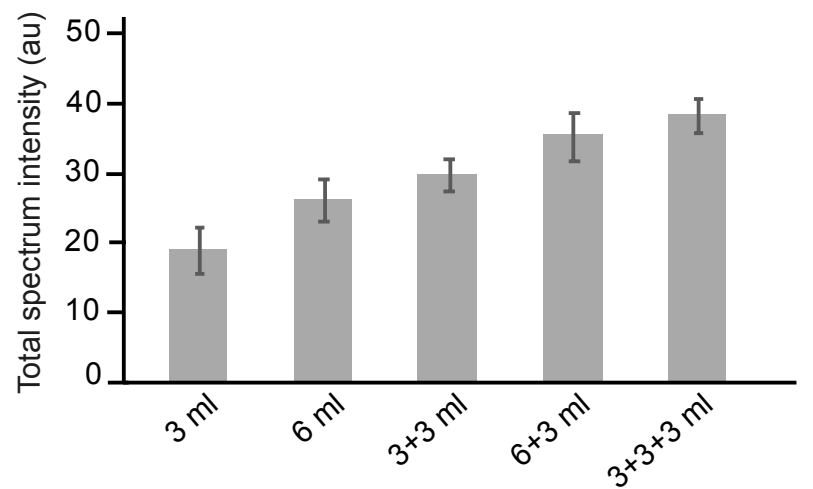

Total wash

volume

$3 \mathrm{ml}$

$6 \mathrm{ml}$

$9 \mathrm{ml}$

$\mathrm{N}^{\circ}$ of washes 1 wash 2 washes 3 washes

(b)

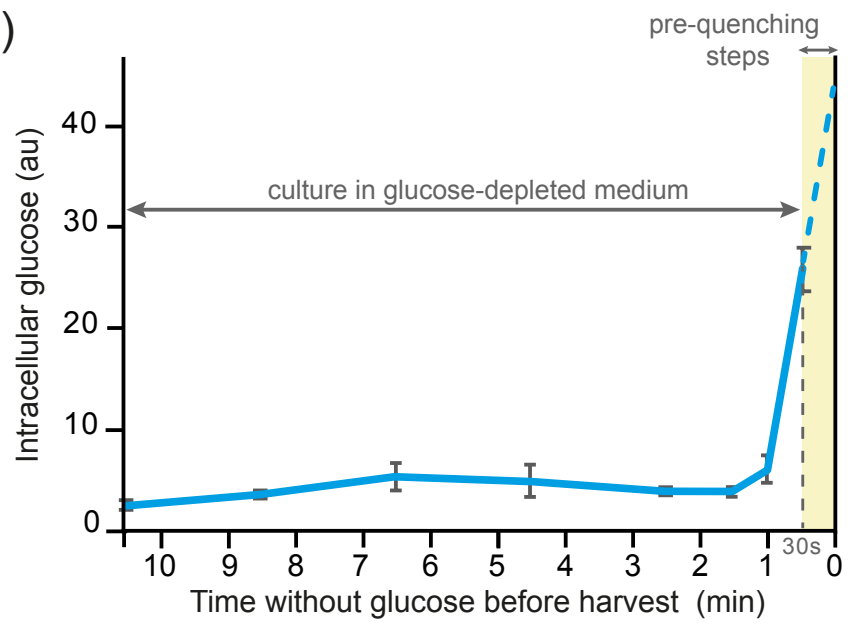


Figure 4

a) $\begin{aligned} & \square \text { Extraction with methanol without cell scraping } \\ & \square \text { Extraction with methanol and cell harvesting by scraping } \\ & \square \text { Extraction with methanol/water and cell harvesting by scraping }\end{aligned}$

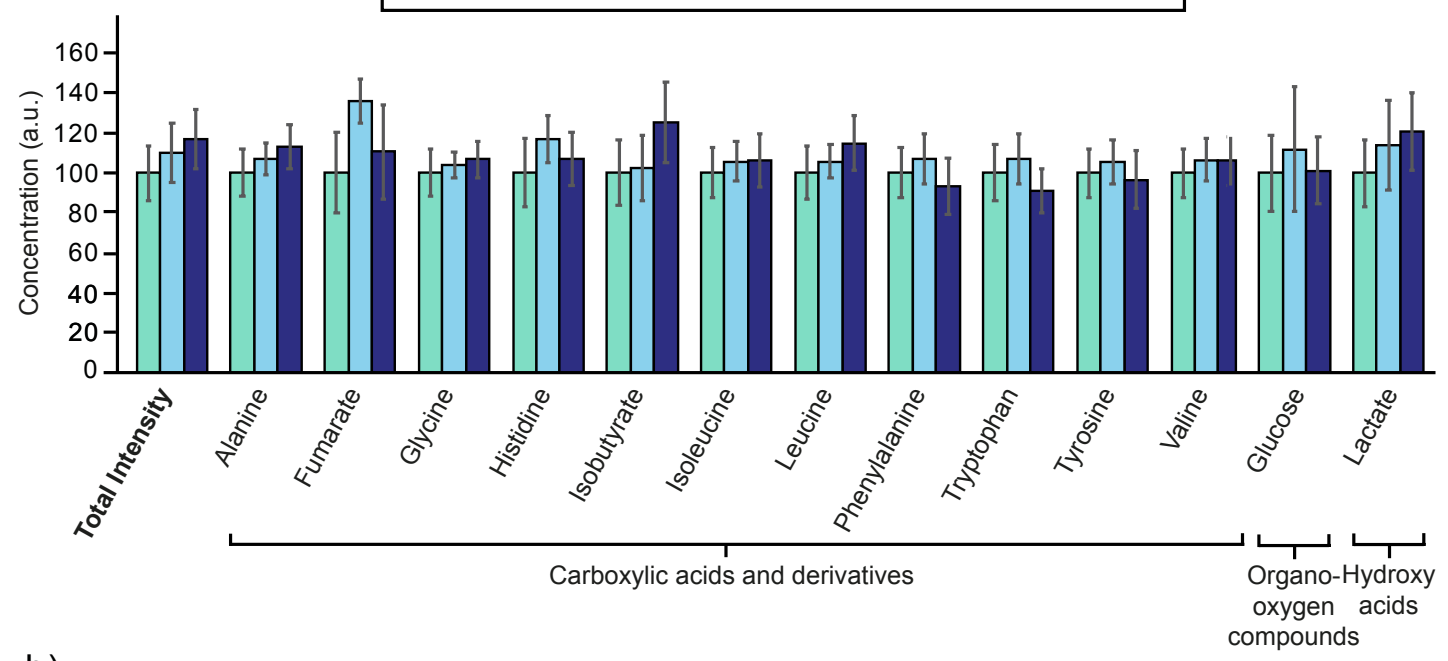

b)

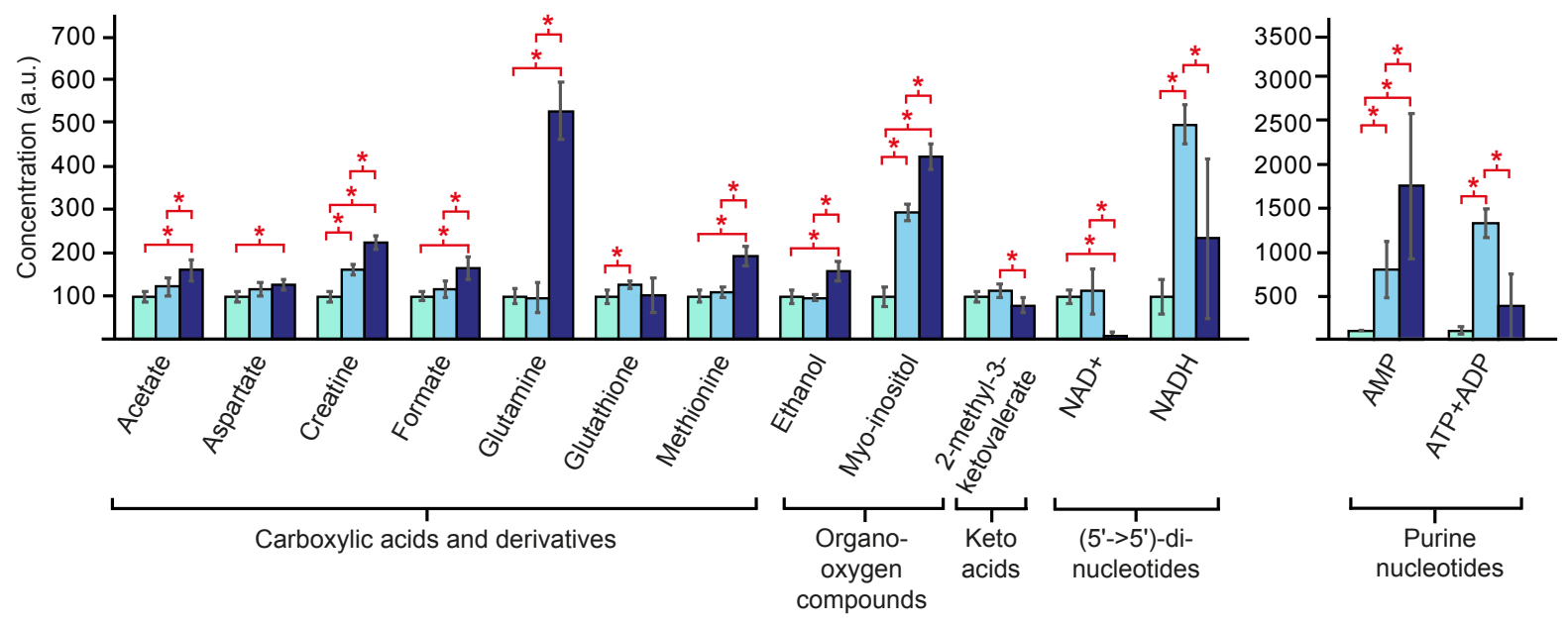




\section{Figure 5}

(a)

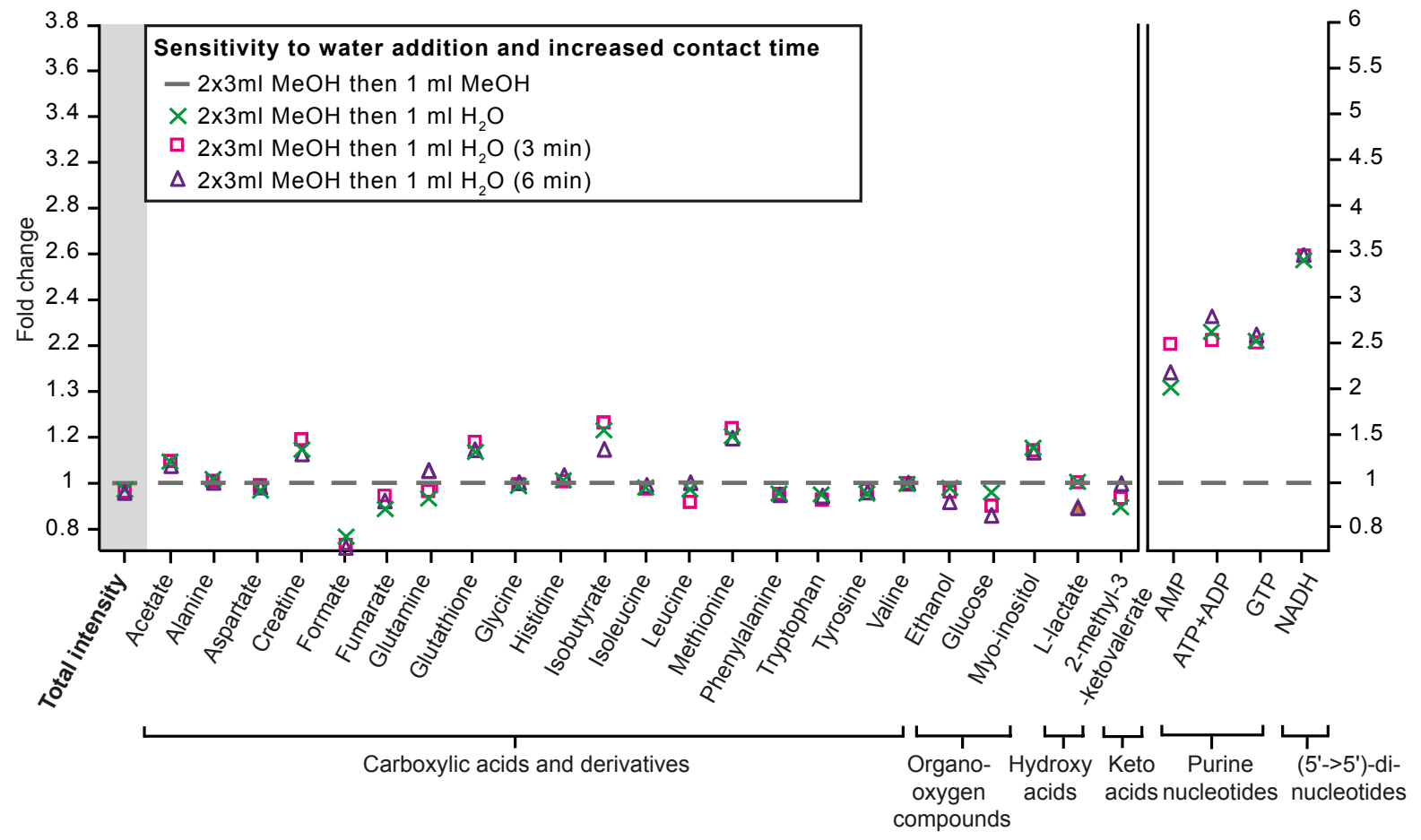

(b)

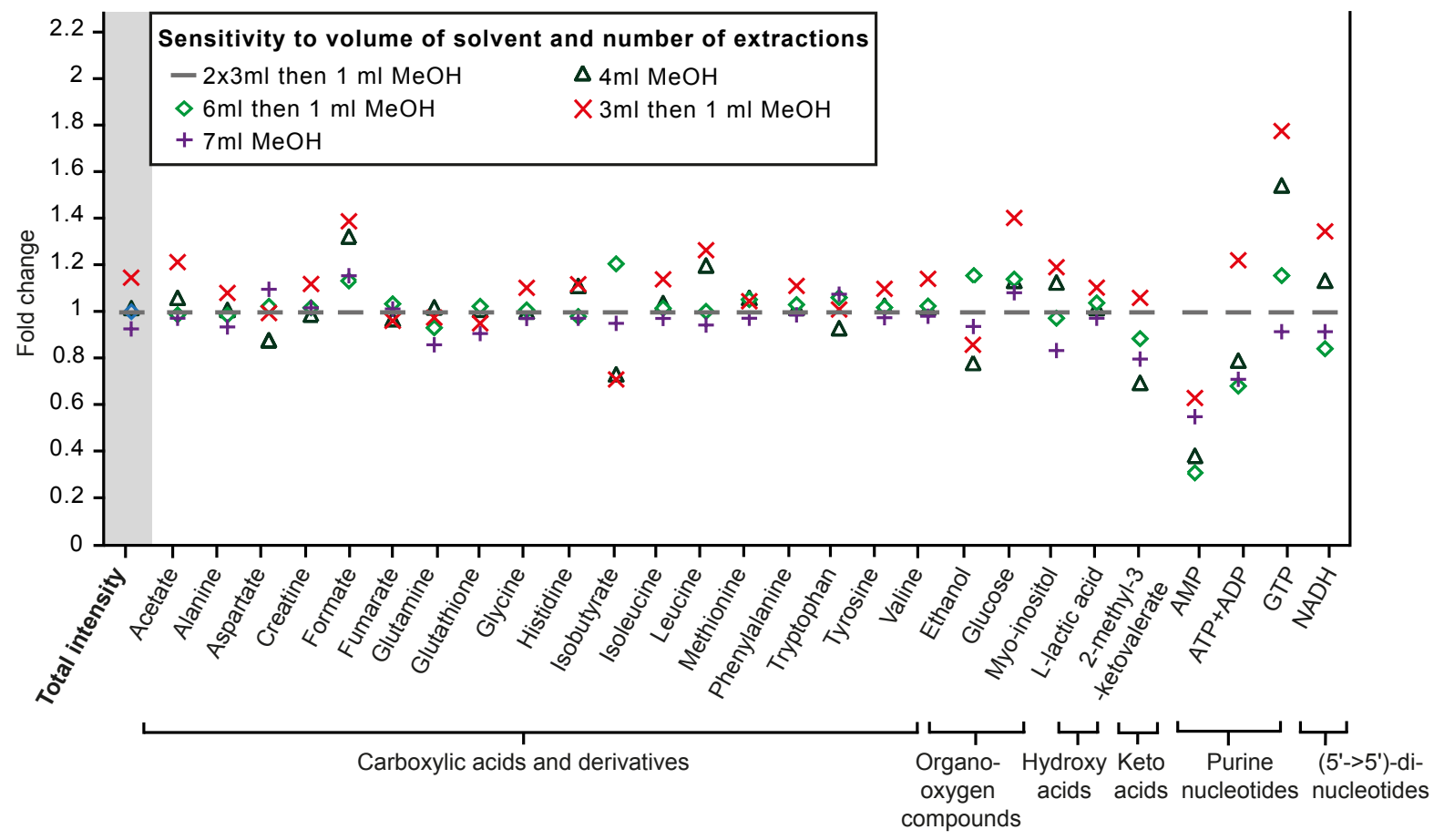

